\title{
Reflected BSDEs Driven by Lévy Processes and Countable Brownian Motions
}

\section{Jean-Marc Owo}

UFR de Mathématiques et Informatique, Université Félix H. Boigny, Abidjan, Côte d'Ivoire

Email: marc.owo@univ-fhb.edu.ci,owo_jm@yahoo.fr

Received 25 March 2015; accepted 20 December 2015; published 23 December 2015

Copyright (C) 2015 by author and Scientific Research Publishing Inc.

This work is licensed under the Creative Commons Attribution International License (CC BY). http://creativecommons.org/licenses/by/4.0/

c) (i) Open Access

\section{Abstract}

A new class of reflected backward stochastic differential equations (RBSDEs) driven by Teugels martingales associated with Lévy process and Countable Brownian Motions are investigated. Via approximation, the existence and uniqueness of solution to this kind of RBSDEs are obtained.

\section{Keywords}

Backward Doubly Stochastic Differential Equations, Lévy Processes, Teugels Martingales, Countable Brownian Motions

\section{Introduction}

Recently, Y. Ren [1] proved via the Snell envelope and the fixed point theorem, the existence and uniqueness of a solution for the following RBDSDEs driven by a Lévy process and a extra Brownian motion with Lipschitz coefficients, where the obstacle process is right continuous with left limits (càdlàg):

$$
Y_{t}=\xi+\int_{t}^{T} f\left(s, Y_{s-}, Z_{s}\right) \mathrm{d} s+\int_{t}^{T} g_{j}\left(s, Y_{s-}, Z_{s}\right) \overline{\mathrm{d}}_{s}^{j}+K_{T}-K_{t}-\sum_{i=1}^{\infty} \int_{t}^{T} Z_{s}^{(i)} \mathrm{d} H_{s}^{(i)},
$$

where the $\mathrm{d} H^{i}$ is a forward semi-martingale Itô integrals (see He et al. [2]) and the $\overline{\mathrm{d} B}$ is a backward Itô integral.

Note that, in all the previous works, the equations are driven by finite Brownian motions. In their recent work, Pengju Duan et al. [3] introduced firstly the reflected BDSDEs driven by countable extra Brownian motions:

$$
Y_{t}=\xi+\int_{t}^{T} f\left(s, Y_{s}, Z_{s}\right) \mathrm{d} s+\sum_{j=1}^{\infty} \int_{t}^{T} g_{j}\left(s, Y_{s}, Z_{s}\right) \overleftarrow{\mathrm{d} B}_{s}^{j}+K_{T}-K_{t}-\int_{t}^{T} Z_{s} \mathrm{~d} W_{s}^{(i)},
$$

where the $\mathrm{d} W$ is the standard forward stochastic Itô integral and the $\overline{\mathrm{dB}}^{j}$ is the backward stochastic Itô integral. 
Under the global Lipschitz continuity conditions on the coefficients $f$ and g, they proved via Snell envelope and fixed point theorem, the existence and uniqueness of the solution for RBDSDEs (1.1). Next, J.-M. Owo [4] relaxed the Lipschitz continuity condition on the coefficient $f$ to a continuity with sub linear growth condition and derive the existence of minimal and maximal solutions to RBSDEs (1.1).

Motivated by [1] [3] [4], in this paper, we mainly consider the following RBDSDEs driven by a Lévy process and countable Brownian motions, in which the obstacle process is right continuous with left limits (càdlàg):

$$
Y_{t}=\xi+\int_{t}^{T} f\left(s, Y_{s-}, Z_{s}\right) \mathrm{d} s+\sum_{j=1}^{\infty} \int_{t}^{T} g_{j}\left(s, Y_{s-}, Z_{s}\right) \overline{\mathrm{d}}_{s}^{j}+K_{T}-K_{t}-\sum_{i=1}^{\infty} \int_{t}^{T} Z_{s}^{(i)} \mathrm{d} H_{s}^{(i)} .
$$

The paper is devoted to prove the existence and uniqueness of a solution for RBSDEs driven by a Lévy process and countable Brownian motions.

The paper is organized as follows. In section 2, we give some preliminaries and notations. In section 3, we establish the main results.

\section{Preliminaries and Notations}

Throughout this paper, $T$ is a positive constant and $(\Omega, \mathcal{F}, \mathbf{P})$ is a probability space on which, $\left\{B_{t}^{j}, 0 \leq t \leq T\right\}_{j=1}^{\infty}$ are mutual independent one-dimensional standard Brownian motions and $\left\{L_{t} ; 0 \leq t \leq T\right\}$ be a $\mathbb{R}$-valued pure jump Lévy process of the form $L_{t}=b t+l_{t}$ independent of $\left\{B_{t}^{j} ; 0 \leq t \leq T\right\}$, which correspond to a standard Lévy

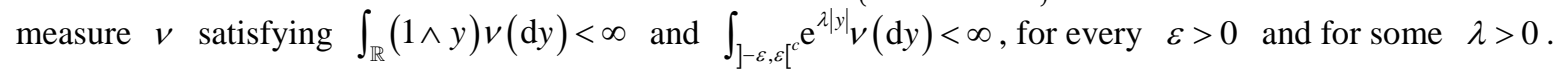

Let $\mathcal{N}$ denote the class of $\mathbf{P}$-null sets of $\mathcal{F}$. For each $t \in[0, T]$, we define

$$
\mathcal{F}_{t} \triangleq\left(\bigvee_{j=1}^{\infty} \mathcal{F}_{t, T}^{B^{j}}\right) \bigvee \mathcal{F}_{t}^{L},
$$

where for any process $\left\{\eta_{t}\right\} ; \mathcal{F}_{s, t}^{\eta}=\sigma\left\{\eta_{r}-\eta_{s} ; s \leq r \leq t\right\} \vee \mathcal{N}, \mathcal{F}_{t}^{\eta}=\mathcal{F}_{0, t}^{\eta}$.

Note that $\left\{\mathcal{F}_{t}^{L}, t \in[0, T]\right\}$ is an increasing filtration and $\left\{\mathcal{F}_{t, T}^{B^{j}}, t \in[0, T]\right\}$ is a decreasing filtration. Thus the collection $\left\{\mathcal{F}_{t}, t \in[0, T]\right\}$ is neither increasing nor decreasing so it does not constitute a filtration.

Let us introduce some spaces:

- $\mathcal{H}_{\mathcal{F}}^{2}$ denotes the space of real-valued processes $\left\{\varphi_{t} ; 0 \leq t \leq T\right\}$ such that $\varphi_{t}$ is $\mathcal{F}_{t}$-measurable, for a.e. $t \in[0, T]$ and $\mathbb{E}\left(\int_{0}^{T}\left|\varphi_{t}\right|^{2} \mathrm{~d} t\right)<\infty$.

- $\quad \mathcal{P}_{\mathcal{F}}^{2}$ denotes the sub set of $\mathcal{H}_{\mathcal{F}}^{2}$ formed by the $\mathcal{F}$-predictable processes;

- $\mathcal{S}_{\mathcal{F}}^{2}$ stands for the set of real-valued, càdàg, random processes $\left\{\varphi_{t} ; 0 \leq t \leq T\right\}$ such that $\varphi_{t}$ is $\mathcal{F}_{t}$ measurable, for any $t \in[0, T]$ and $\|\varphi\|_{\mathcal{S}^{2}}^{2}=\mathbb{E}\left(\sup _{0 \leq t \leq T}\left|\varphi_{t}\right|^{2}\right)<\infty$.

- $\mathcal{A}_{\mathcal{F}}^{2}$ denotes the space continuous, real-valued, increasing processes $\left\{K_{t} ; 0 \leq t \leq T\right\}$, such that $K_{t}$ is $\mathcal{F}_{t}$ measurable, for a.e. $t \in[0, T], \quad K_{0}=0$ and $\mathbb{E}\left(\left|K_{T}\right|^{2}\right)<\infty$.

- $\ell^{2}$ denotes the set of real valued sequences $\left(x_{n}\right)_{n \geq 1}$ such that $\|x\|_{\ell^{2}}^{2}=\sum_{i=1}^{\infty}\left|x_{i}\right|^{2}<\infty$.

We will denote by $\mathcal{H}_{\mathcal{F}}^{2}\left(\ell^{2}\right)$ and $\mathcal{P}_{\mathcal{F}}^{2}\left(\ell^{2}\right)$ the corresponding spaces of $\ell^{2}$-valued processes $\left\{\varphi_{t} ; 0 \leq t \leq T\right\}$ such that

$$
\|\varphi\|_{\mathcal{M}^{2}\left(\ell^{2}\right)}^{2}=\mathbb{E}\left(\int_{0}^{T}\left\|\varphi_{t}\right\|_{\ell^{2}}^{2} \mathrm{~d} t\right)=\sum_{i=1}^{\infty} \mathbb{E}\left(\int_{0}^{T}\left|\varphi_{t}^{(i)}\right|^{2} \mathrm{~d} t\right)<\infty
$$

In the sequel, for ease of notation, we set $\|\cdot\|_{\ell^{2}}=\|\cdot\|$.

Furthermore, we denote by $\left(H^{(i)}\right)_{i \geq 1}$ the Teugels Martingale associated with the Lévy process $\left\{L_{t} ; 0 \leq t \leq T\right\}$. More precisely

$$
H_{t}^{(i)}=c_{i, i} T_{t}^{(i)}+c_{i, i-1} T_{t}^{(i-1)}+\cdots+c_{i, 1} T_{t}^{(1)},
$$

where $T_{t}^{(i)}=L_{t}^{(i)}-\mathbf{E}\left(L_{t}^{(i)}\right)=L_{t}^{(i)}-t \mathbf{E}\left(L_{1}^{(i)}\right)$ for all $i \geq 1$ and $L_{t}^{(i)}$ are power-jump processes. That is, $L_{t}^{(1)}=L_{t}$ and $L_{t}^{(i)}=\sum_{0<s \leq t}\left(\Delta L_{s}\right)^{i}$ for $i \geq 2$, with $\Delta L_{s}=L_{s}-L_{s-}$. 
In [5], Nualart and Schoutens proved that the coefficients $c_{i, k}$ correspond to the orthonormalization of the polynomials $1, x, x^{2}, \cdots$ with respect to the measure $\mu(\mathrm{d} x)=x^{2} v(\mathrm{~d} x)+\sigma^{2} \delta_{0}(\mathrm{~d} x)$, i.e.

$q_{i}(x)=c_{i, i} x^{i-1}+c_{i, i-1} x^{i-2}+\cdots+c_{i, 1}$. The martingale $\left(H^{(i)}\right)$ can be chosen to be pairwise strongly orthonormal martingale. That is, for all $i, j,\left\langle H^{(i)}, H^{(j)}\right\rangle=\delta_{i j}$.

Definition 2.1. A solution of a (1.2) is a triplet of $\left(\mathbb{R} \times \ell^{2} \times \mathbb{R}_{+}\right)$-valued process $(Y, Z, K)$, which satisfies (1.2), and

1) $(Y, Z, K) \in \mathcal{S}_{\mathcal{F}}^{2} \times \mathcal{P}_{\mathcal{F}}^{2}\left(\ell^{2}\right) \times \mathcal{A}_{\mathcal{F}}^{2}$;

2) $Y_{t} \geq S_{t}, \forall t \in[0, T]$;

3) $K$ is a continuous and increasing process with $K_{0}=0$ and $\int_{0}^{T}\left(Y_{t}-S_{t}\right) \mathrm{d} K_{t}=0$.

Throughout the paper, we let the coefficients $f: \Omega \times[0, T] \times \mathbb{R} \times \ell^{2} \rightarrow \mathbb{R}$ and $g_{j}: \Omega \times[0, T] \times \mathbb{R} \times \ell^{2} \rightarrow \mathbb{R}$, the terminal value $\xi: \Omega \rightarrow \mathbb{R}$ and the obstacle $S: \Omega \times[0, T] \rightarrow \mathbb{R}$ satisfying the following assumptions:

(H1) for all $(t, y, z) \in[0, T] \times \mathbb{R} \times \ell^{2}, f(t, y, z),\left\{g_{j}(t, y, z)\right\}_{j=1}^{\infty}$ are $\mathcal{F}_{t}$-measurable such that

$$
\mathbf{E} \int_{0}^{T}|f(s, 0,0)|^{2} \mathrm{~d} s+\sum_{j=1}^{\infty} \mathbf{E} \int_{0}^{T}\left|g_{j}(s, 0,0)\right|^{2} \mathrm{~d} s<+\infty ;
$$

(H2) for all $t \in[0, T]$ and $\left(y_{1}, z_{1}\right),\left(y_{2}, z_{2}\right) \in \mathbb{R} \times \ell^{2}$,

$$
\left\{\begin{array}{l}
\left|f\left(t, y_{1}, z_{1}\right)-f\left(t, y_{2}, z_{2}\right)\right|^{2} \leq C\left(\left|y_{1}-y_{2}\right|^{2}+\left\|z_{1}-z_{2}\right\|^{2}\right) \\
\left|g_{j}\left(t, y_{1}, z_{1}\right)-g_{j}\left(t, y_{2}, z_{2}\right)\right|^{2} \leq C_{j}\left|y_{1}-y_{2}\right|^{2}+\alpha_{j}\left\|z_{1}-z_{2}\right\|^{2}
\end{array}\right.
$$

where $C>0, C_{j}>0$ and $\alpha_{j}>0$ are constants with $\sum_{j=1}^{\infty} C_{j}<\infty$ and $\alpha=\sum_{j=1}^{\infty} \alpha_{j}<1$.

(H3) $\xi \in L^{2}\left(\Omega, \mathcal{F}_{T}, \mathbf{P}\right)$, i.e. $\xi$ is a $\mathcal{F}_{t}$-measurable random variable such that, $\mathbf{E}\left(|\xi|^{2}\right)<\infty$,

(H4) $S$ is a real-valued, càdàg process such that $S_{t}$ is $\mathcal{F}_{t}$-measurable, for a.e. $t \in[0, T]$ and $S_{T} \leq \xi$ a.s., with $\mathbb{E}\left(\sup _{0 \leq t \leq T}\left(S_{t}^{+}\right)^{2}\right)<\infty$, where $S_{t}^{+}=\max \left(S_{t}, 0\right)$. Moreover, we assume that its jumping times are inaccessible stopping times (see He et al. [2]).

\section{The Main Results}

We first establish the existence and uniqueness result for RBSDEs driven by finite Brownian motions and a Lévy process:

$$
Y_{t}=\xi+\int_{t}^{T} f\left(s, Y_{s-}, Z_{s}\right) \mathrm{d} s+\sum_{j=1}^{n} \int_{t}^{T} g_{j}\left(s, Y_{s-}, Z_{s}\right) \overline{\mathrm{d}}_{s}^{j}+K_{T}-K_{t}-\sum_{i=1}^{\infty} \int_{t}^{T} Z_{s}^{(i)} \mathrm{d} H_{s}^{(i)}
$$

For any $n \geq 1$, we have the following existence and uniqueness result.

Lemma 3.2. Assume (H1) - (H4). Then, there exists a unique solution ( $Y, Z, K)$ of Equation (3.1).

Proof. For $n=1$, we obtain the existence and uniqueness result due to Y. Ren [1]. For any $n>1$, we can prove the desired result following the same ideas and arguments as in Y. Ren [1]: it is a straightforward adaptation of the proofs of Theorem 2 and Theorem 3 in Y. Ren [1]. Firstly, we consider the special case that is the function $f$ and $g_{j}$ do not depend on $(Y, Z)$, i.e. $f(\omega, t, y, z) \equiv f(\omega, t), g_{j}(\omega, t, y, z) \equiv g_{j}(\omega, t)$, for all $(\omega, t, y, z) \in \Omega \times[0, T] \times \mathbb{R} \times \ell^{2}$. It suffices to replace suitably $\mathcal{F}_{0, T}^{B}$ and $\int_{t}^{T} g(s) \mathrm{d} B_{s}$ in the proof of Theorem 2 respectively by $\bigvee_{j=1}^{n} \mathcal{F}_{0, T}^{B^{j}}$ and $\sum_{j=1}^{n} \int_{t}^{T} g_{j}(s) \overline{\mathrm{d} B}_{s}^{j}$. On the other hand, it suffices to replace

$\int_{t}^{T} \mathrm{e}^{\beta s}\left(Y_{s}-Y_{s}^{\prime}\right)\left(g\left(s, \tilde{Y}_{s-}, \tilde{Z}_{s}\right)-g\left(s, \tilde{Y}_{s-}^{\prime}, \tilde{Z}_{s}^{\prime}\right)\right) \mathrm{d} B_{s}, \int_{t}^{T} \mathrm{e}^{\beta s}\left|g\left(s, \tilde{Y}_{s-}, \tilde{Z}_{s}\right)-g\left(s, \tilde{Y}_{s-}^{\prime}, \tilde{Z}_{s}^{\prime}\right)\right|^{2} \mathrm{~d} s, C$ and $\alpha$ in the proof of Theorem 3 respectively by $\sum_{j=1}^{n} \int_{t}^{T} \mathrm{e}^{\beta s}\left(Y_{s}-Y_{s}^{\prime}\right)\left(g_{j}\left(s, \tilde{Y}_{s-}, \tilde{Z}_{s}\right)-g_{j}\left(s, \tilde{Y}_{s-}^{\prime}, \tilde{Z}_{s}^{\prime}\right)\right){\overleftarrow{\mathrm{d}} B_{s}^{j}}^{j}$, $\sum_{j=1}^{n} \int_{t}^{T} \mathrm{e}^{\beta s}\left|g_{j}\left(s, \tilde{Y}_{s-}, \tilde{Z}_{s}\right)-g_{j}\left(s, \tilde{Y}_{s-}^{\prime}, \tilde{Z}_{s}^{\prime}\right)\right|^{2} \mathrm{~d} s, \sum_{j=1}^{n} C_{j}$ and $\sum_{j=1}^{n} \alpha_{j}$. Therefore, we omit the details.

Now, we are ready to establish the main result of this paper which is the following theorem.

Theorem 3.3. Under assumptions (H1)-(H4), there exists a unique solution $(Y, Z, K) \in \mathcal{S}_{\mathcal{F}}^{2} \times \mathcal{P}_{\mathcal{F}}^{2}\left(\ell^{2}\right) \times \mathcal{A}_{\mathcal{F}}^{2}$ of Equation (1.2). 
Proof. (Existence.) By Lemma 3.1, for any $n \geq 1$, there exists a unique solution of (3.1), denoted by $\left(Y^{n}, Z^{n}, K^{n}\right)$, i.e., $\left(Y^{n}, Z^{n}, K^{n}\right) \in \mathcal{S}_{\mathcal{F}}^{2} \times \mathcal{P}_{\mathcal{F}}^{2}\left(\ell^{2}\right) \times \mathcal{A}_{\mathcal{F}}^{2}$ and

$$
\begin{aligned}
& \text { 1) } Y_{t}^{n}=\xi+\int_{t}^{T} f\left(s, Y_{s-}^{n}, Z_{s}^{n}\right) \mathrm{d} s+\sum_{j=1}^{n} \int_{t}^{T} g_{j}\left(s, Y_{s-}^{n}, Z_{s}^{n}\right) \overline{\mathrm{d}}_{s}^{j}+K_{T}^{n}-K_{t}^{n}-\sum_{i=1}^{\infty} \int_{t}^{T} Z_{s}^{n(i)} \mathrm{d} H_{s}^{(i)} ; \\
& \text { 2) } Y_{t}^{n} \geq S_{t}, \quad \forall t \in[0, T] \text { and } \int_{0}^{T}\left(Y_{t}^{n}-S_{t}\right) \mathrm{d} K_{t}^{n}=0 .
\end{aligned}
$$

The idea consists to study the convergence of the sequence $\left(Y^{n}, Z^{n}, K^{n}\right)$, and to establish that its limit is a solution of (1.2). To this end, we first establish the following estimates:

$$
\sup _{n \geq 1} \mathbf{E}\left(\sup _{0 \leq t \leq T}\left|Y_{t}^{n}\right|^{2}+\int_{0}^{T}\left|Z_{t}^{n}\right|^{2} \mathrm{~d} t+\left|K_{T}^{n}\right|^{2}\right) \leq \lambda,
$$

where $\lambda$ is a non-negative constant independent of $n$. Indeed, applying Itô's formula to $\left|Y_{t}^{n}\right|^{2}$, we have

$$
\mathbf{E}\left|Y_{t}^{n}\right|^{2}+\mathbf{E} \int_{t}^{T}\left\|Z_{s}^{n}\right\|^{2} \mathrm{~d} s=\mathbf{E}|\xi|^{2}+2 \mathbf{E} \int_{t}^{T} Y_{s}^{n} f\left(s, Y_{s}^{n}, Z_{s}^{n}\right) \mathrm{d} s+2 \mathbf{E} \int_{t}^{T} Y_{s}^{n} \mathrm{~d} K_{s}^{n}+\sum_{j=1}^{n} \mathbf{E} \int_{t}^{T}\left|g_{j}\left(s, Y_{s}^{n}, Z_{s}^{n}\right)\right|^{2} \mathrm{~d} s .
$$

From assumption (H2) and Young's inequality, for any $\theta>0$, we have

$$
\begin{gathered}
2 Y_{s}^{n} f\left(s, Y_{s}^{n}, Z_{s}^{n}\right) \leq \frac{2 C}{\theta}\left|Y_{s}^{n}\right|^{2}+\frac{\theta}{2 C}\left|f\left(s, Y_{s}^{n}, Z_{s}^{n}\right)\right|^{2} \leq\left(\frac{2 C}{\theta}+\theta\right)\left|Y_{s}^{n}\right|^{2}+\theta \| Z_{s}^{n}||^{2}+\frac{\theta}{C}|f(s, 0,0)|^{2}, \\
\left|g_{j}\left(s, Y_{s}^{n}, Z_{s}^{n}\right)\right|^{2} \leq(1+\theta) C_{j}\left|Y_{s}^{n}\right|^{2}+(1+\theta) \alpha_{j}\left\|Z_{s}^{n}\right\|^{2}+\left(1+\frac{1}{\theta}\right)\left|g_{j}(s, 0,0)\right|^{2} .
\end{gathered}
$$

Using again Young inequality, we have for any $\beta>0$,

$$
2 \mathbf{E} \int_{t}^{T} Y_{s}^{n} \mathrm{~d} K_{s}^{n}=2 \mathbf{E} \int_{t}^{T} S_{s} \mathrm{~d} K_{s}^{n} \leq \frac{1}{\beta} \mathbf{E} \sup _{0 \leq s \leq T}\left|S_{s}\right|^{2}+\beta \mathbf{E}\left(K_{T}^{n}-K_{t}^{n}\right)^{2} .
$$

Since

$$
K_{T}^{n}-K_{t}^{n}=Y_{t}^{n}-\xi-\int_{t}^{T} f\left(s, Y_{s}^{n}, Z_{s}^{n}\right) \mathrm{d} s-\sum_{j=1}^{n} \int_{t}^{T} g_{j}\left(s, Y_{s}^{n}, Z_{s}^{n}\right) \overline{\mathrm{d}}_{s}^{j}+\sum_{i=1}^{\infty} \int_{t}^{T} Z_{s}^{n(i)} \mathrm{d} H_{s}^{(i)}, t \in[0, T],
$$

we have, for any $t \in[0, T]$,

$$
\begin{aligned}
\mathbf{E}\left(K_{T}^{n}-K_{t}^{n}\right)^{2} \leq & 5 \mathbf{E}\left(\left|Y_{t}^{n}\right|^{2}+|\xi|^{2}+\left|\int_{t}^{T} f\left(s, Y_{s}^{n}, Z_{s}^{n}\right) \mathrm{d} s\right|^{2}+\left.\left|\sum_{j=1}^{n} \int_{t}^{T} g_{j}\left(s, Y_{s}^{n}, Z_{s}^{n}\right) \overline{\mathrm{d} B}\right|_{s}^{j}\right|^{2}+\left|\sum_{i=1}^{\infty} \int_{t}^{T} Z_{s}^{n(i)} \mathrm{d} H_{s}^{(i)}\right|^{2}\right) \\
\leq & 5 \mathbf{E}\left(\left|Y_{t}^{n}\right|^{2}+|\xi|^{2}+2 T \int_{t}^{T}\left(C\left|Y_{s}^{n}\right|^{2}+C\left|Z_{s}^{n}\right|^{2}+|f(s, 0,0)|^{2}\right) \mathrm{d} s\right) \\
& +5 \mathbf{E}\left(\sum_{j=1}^{\infty} \int_{t}^{T}\left((1+\theta) C_{j}\left|Y_{s}^{n}\right|^{2}+(1+\theta) \alpha_{j} \|\left. Z_{s}^{n}\right|^{2}+\left(1+\frac{1}{\theta}\right)\left|g_{j}(s, 0,0)\right|^{2}\right) \mathrm{d} s+\int_{t}^{T}\left|Z_{s}^{n}\right|^{2} \mathrm{~d} s\right),
\end{aligned}
$$

Therefore,

$$
\begin{aligned}
& \mathbf{E}\left|Y_{t}^{n}\right|^{2}+\mathbf{E} \int_{t}^{T}\left\|Z_{s}^{n}\right\|^{2} \mathrm{~d} s \leq \mathbf{E}|\xi|^{2}+\mathbf{E} \int_{t}^{T}\left(\left(\frac{2 C}{\theta}+\theta\right)\left|Y_{s}^{n}\right|^{2}+\theta\left|Z_{s}^{n}\right|^{2}+\frac{\theta}{C}|f(s, 0,0)|^{2}\right) \mathrm{d} s+\frac{1}{\beta} \mathbf{E} \sup _{0 \leq s \leq T}\left|S_{s}\right|^{2} \\
& \quad 5 \beta \mathbf{E}\left(\left|Y_{t}^{n}\right|^{2}+|\xi|^{2}+2 T \int_{t}^{T}\left(C\left|Y_{s}^{n}\right|^{2}+C\left\|Z_{s}^{n}\right\|^{2}+|f(s, 0,0)|^{2}\right) \mathrm{d} s\right) \\
& +5 \beta \sum_{j=1}^{\infty} \mathbf{E} \int_{t}^{T}\left((1+\theta) C_{j}\left|Y_{s}^{n}\right|^{2}+(1+\theta) \alpha_{j}\left\|Z_{s}^{n}\right\|^{2}+\left(1+\frac{1}{\theta}\right)\left|g_{j}(s, 0,0)\right|^{2}\right) \mathrm{d} s+5 \beta \mathbf{E} \int_{t}^{T}\left\|Z_{s}^{n}\right\|^{2} \mathrm{~d} s \\
& \quad+\sum_{j=1}^{\infty} \mathbf{E} \int_{t}^{T}\left((1+\theta) C_{j}\left|Y_{s}^{n}\right|^{2}+(1+\theta) \alpha_{j}\left\|Z_{s}^{n}\right\|^{2}+\left(1+\frac{1}{\theta}\right)\left|g_{j}(s, 0,0)\right|^{2}\right) \mathrm{d} s .
\end{aligned}
$$


Consequently,

$$
\begin{aligned}
& (1-5 \beta) \mathbf{E}\left|Y_{t}^{n}\right|^{2}+[1-\theta-(1+\theta) \alpha-5 \beta(2 T C+(1+\theta) \alpha+1)] \mathbf{E} \int_{t}^{T}\left\|Z_{s}^{n}\right\|^{2} \mathrm{~d} s \\
& \leq(1+5 \beta) \mathbf{E}|\xi|^{2}+\left[\left(\frac{2 C}{\theta}+\theta\right)+(5 \beta+1)(1+\theta) \sum_{j=1}^{\infty} C_{j}+10 \beta T C\right] \mathbf{E} \int_{t}^{T}\left|Y_{s}^{n}\right|^{2} \mathrm{~d} s \\
& \quad+\left(\frac{C}{\theta}+10 \beta T\right) \mathbf{E} \int_{t}^{T}|f(s, 0,0)|^{2} \mathrm{~d} s+\left(1+\frac{1}{\theta}\right)(1+5 \beta) \sum_{j=1}^{\infty} \mathbf{E} \int_{t}^{T}\left|g_{j}(s, 0,0)\right|^{2} \mathrm{~d} s+\frac{1}{\beta} \mathbf{E} \sup _{0 \leq s \leq T}\left|S_{s}\right|^{2}
\end{aligned}
$$

We choose $\beta, \theta>0$ such that, $\beta<\frac{1-\alpha}{5(2 T C+\alpha+1)}, \theta \leq \frac{1-\alpha-5 \beta(2 T C+\alpha+1)}{1+\alpha+5 \beta \alpha}$. Then, there exists a constant $c=c(\alpha, T, C)>0$, such that

$$
\mathbf{E}\left|Y_{t}^{n}\right|^{2} \leq c \mathbf{E}\left(|\xi|^{2}+\int_{t}^{T}\left|Y_{s}^{n}\right|^{2} \mathrm{~d} s+\int_{t}^{T}|f(s, 0,0)|^{2} \mathrm{~d} s+\sum_{j=1}^{\infty} \int_{t}^{T}\left|g_{j}(s, 0,0)\right|^{2} \mathrm{~d} s+\sup _{0 \leq s \leq T}\left|S_{s}\right|^{2}\right),
$$

Applying Gronwall’s inequality, we get

$$
\mathbf{E}\left|Y_{t}^{n}\right|^{2} \leq c \mathrm{e}^{c T} \mathbf{E}\left(|\xi|^{2}+\int_{t}^{T}|f(s, 0,0)|^{2} \mathrm{~d} s+\sum_{j=1}^{\infty} \int_{t}^{T}\left|g_{j}(s, 0,0)\right|^{2} \mathrm{~d} s+\sup _{0 \leq s \leq T}\left|S_{s}\right|^{2}\right) .
$$

Therefore, we have the existence of a constant $c_{1}$ such that

$$
\begin{aligned}
& \mathbf{E}\left(\left|Y_{t}^{n}\right|^{2}+\int_{t}^{T} \| Z_{s}^{n}||^{2} \mathrm{~d} s+\left|K_{T}^{n}\right|^{2}\right) \\
& \leq c_{1} \mathbf{E}\left(|\xi|^{2}+\int_{t}^{T}|f(s, 0,0)|^{2} \mathrm{~d} s+\sum_{j=1}^{\infty} \int_{t}^{T}\left|g_{j}(s, 0,0)\right|^{2} \mathrm{~d} s+\sup _{0 \leq s \leq T}\left|S_{s}\right|^{2}\right),
\end{aligned}
$$

which by Burkhölder-Davis-Gundy’s inequality provides

$$
\begin{aligned}
& \mathbf{E}\left(\sup _{0 \leq t \leq T}\left|Y_{t}^{n}\right|^{2}+\int_{0}^{T}\left\|Z_{s}^{n}\right\|^{2} \mathrm{~d} s+\left|K_{T}^{n}\right|^{2}\right) \\
& \leq c_{1} \mathbf{E}\left(|\xi|^{2}+\int_{0}^{T}|f(s, 0,0)|^{2} \mathrm{~d} s+\sum_{j=1}^{\infty} \int_{0}^{T}\left|g_{j}(s, 0,0)\right|^{2} \mathrm{~d} s+\sup _{0 \leq s \leq T}\left|S_{s}\right|^{2}\right)<\infty .
\end{aligned}
$$

Now, we show that $\left(Y^{n}, Z^{n}, K^{n}\right)$ is a Cauchy sequence in $\mathcal{S}_{\mathcal{F}}^{2} \times \mathcal{P}_{\mathcal{F}}^{2}\left(\ell^{2}\right) \times \mathcal{A}_{\mathcal{F}}^{2}$. To this end, without loss of generality, we let $m<n$. Then, by difference, we obtain

$$
\begin{aligned}
Y_{t}^{n}-Y_{t}^{m}= & \int_{t}^{T}\left(f\left(s, Y_{s-}^{n}, Z_{s}^{n}\right)-f\left(s, Y_{s-}^{m}, Z_{s}^{m}\right)\right) \mathrm{d} s+\sum_{j=1}^{m} \int_{t}^{T}\left(g_{j}\left(s, Y_{s-}^{n}, Z_{s}^{n}\right)-g_{j}\left(s, Y_{s-}^{m}, Z_{s}^{m}\right)\right) \mathrm{d} B_{s}^{j} \\
& +\sum_{j=m+1}^{n} \int_{t}^{T} g_{j}\left(s, Y_{s-}^{n}, Z_{s}^{n}\right) \mathrm{d} B_{s}^{j}+\int_{t}^{T}\left(\mathrm{~d} K_{s}^{n}-\mathrm{d} K_{s}^{m}\right)-\sum_{i=1}^{\infty} \int_{t}^{T}\left(Z_{s}^{n(i)}-Z_{s}^{m(i)}\right) \mathrm{d} H_{s}^{(i)} .
\end{aligned}
$$

Applying Itô's formula to $\left|Y_{t}^{n}-Y_{t}^{m}\right|^{2}$, we get

$$
\begin{aligned}
\left|Y_{t}^{n}-Y_{t}^{m}\right|^{2}= & 2 \int_{t}^{T}\left(Y_{s}^{n}-Y_{s}^{m}\right)\left(f\left(s, Y_{s-}^{n}, Z_{s}^{n}\right)-f\left(s, Y_{s-}^{m}, Z_{s}^{m}\right)\right) \mathrm{d} s+2 \sum_{j=m+1}^{n} \int_{t}^{T}\left(Y_{s}^{n}-Y_{s}^{m}\right) g_{j}\left(s, Y_{s-}^{n}, Z_{s}^{n}\right) \mathrm{d} B_{s}^{j} \\
& +2 \sum_{j=1}^{m} \int_{t}^{T}\left(Y_{s}^{n}-Y_{s}^{m}\right)\left(g_{j}\left(s, Y_{s-}^{n}, Z_{s}^{n}\right)-g_{j}\left(s, Y_{s-}^{m}, Z_{s}^{m}\right)\right) \mathrm{d} B_{s}^{j}+2 \int_{t}^{T}\left(Y_{s}^{n}-Y_{s}^{m}\right)\left(\mathrm{d} K_{s}^{n}-\mathrm{d} K_{s}^{m}\right) \\
& -2 \sum_{i=1}^{\infty} \int_{t}^{T}\left(Y_{s}^{n}-Y_{s}^{m}\right)\left(Z_{s}^{n(i)}-Z_{s}^{m(i)}\right) \mathrm{d} H_{s}^{(i)}+\sum_{j=1}^{m} \int_{t}^{T}\left|g_{j}\left(s, Y_{s-}^{n}, Z_{s}^{n}\right)-g_{j}\left(s, Y_{s-}^{m}, Z_{s}^{m}\right)\right|^{2} \mathrm{~d} s \\
& +\sum_{j=m+1}^{n} \int_{t}^{T}\left|g_{j}\left(s, Y_{s-}^{n}, Z_{s}^{n}\right)\right|^{2} \mathrm{~d} s-\sum_{i, j=1}^{\infty} \int_{t}^{T}\left(Z_{s}^{n(i)}-Z_{s}^{m(i)}\right)\left(Z_{s}^{n(j)}-Z_{s}^{m(j)}\right) \mathrm{d}\left[H^{(i)}, H^{(j)}\right]_{s} .
\end{aligned}
$$


Taking expectation in both side of (3.5) and noting that $\int_{t}^{T}\left(Y_{s}^{n}-Y_{s}^{m}\right)\left(\mathrm{d} K_{s}^{n}-\mathrm{d} K_{s}^{m}\right) \leq 0$, we have

$$
\begin{aligned}
& \mathbf{E}\left(\left|Y_{t}^{n}-Y_{t}^{m}\right|^{2}+\int_{t}^{T}\left\|Z_{s}^{n}-Z_{s}^{m}\right\|^{2} \mathrm{~d} s\right) \\
& \leq 2 \mathbf{E} \int_{t}^{T}\left(Y_{s}^{n}-Y_{s}^{m}\right)\left(f\left(s, Y_{s-}^{n}, Z_{s}^{n}\right)-f\left(s, Y_{s-}^{m}, Z_{s}^{m}\right)\right) \mathrm{d} s+\sum_{j=m+1}^{n} \mathbf{E} \int_{t}^{T}\left|g_{j}\left(s, Y_{s-}^{n}, Z_{s}^{n}\right)\right|^{2} \mathrm{~d} s \\
& \quad+\sum_{j=1}^{m} \mathbf{E} \int_{t}^{T}\left|g_{j}\left(s, Y_{s-}^{n}, Z_{s}^{n}\right)-g_{j}\left(s, Y_{s-}^{m}, Z_{s}^{m}\right)\right|^{2} \mathrm{~d} s .
\end{aligned}
$$

Using again Young's inequality, assumption (H2) and the estimates (3.3), we obtain,

$$
\begin{aligned}
& \mathbf{E}\left(\left|Y_{t}^{n}-Y_{t}^{m}\right|^{2}+\frac{1-\alpha}{2} \int_{t}^{T}\left\|Z_{s}^{n}-Z_{s}^{m}\right\|^{2} \mathrm{~d} s\right) \\
& \leq C_{p} \mathbf{E} \int_{t}^{T}\left|Y_{s}^{n}-Y_{s}^{m}\right|^{2} \mathrm{~d} s+2 \sum_{j=m+1}^{n}\left(\lambda T C_{j}+\lambda \alpha_{j}+\mathbf{E} \int_{0}^{T}\left|g_{j}(s, 0,0)\right|^{2} \mathrm{~d} s\right),
\end{aligned}
$$

where $C_{p}=\frac{2 C}{1-\alpha}+\sum_{j=1}^{\infty} C_{j}+\frac{1-\alpha}{2}$.

Therefore, by Gronwall's inequality, we have

$$
\mathbf{E}\left(\left|Y_{t}^{n}-Y_{t}^{m}\right|^{2}+\int_{t}^{T}\left\|Z_{s}^{n}-Z_{s}^{m}\right\|^{2} \mathrm{~d} s\right) \leq 2 \mathrm{e}^{C_{p} T} \sum_{j=m+1}^{n}\left(\lambda T C_{j}+\lambda \alpha_{j}+\mathbf{E} \int_{0}^{T}\left|g_{j}(s, 0,0)\right|^{2} \mathrm{~d} s\right),
$$

which, by Burkholder-Davis-Gundy inequality provides

$$
\mathbf{E}\left(\sup _{0 \leq t \leq T}\left|Y_{t}^{n}-Y_{t}^{m}\right|^{2}+\int_{0}^{T}|| Z_{s}^{n}-\left.Z_{s}^{m}\right|^{2} \mathrm{~d} s\right) \leq c \sum_{j=m+1}^{n}\left(\lambda T C_{j}+\lambda \alpha_{j}+\mathbf{E} \int_{0}^{T}\left|g_{j}(s, 0,0)\right|^{2} \mathrm{~d} s\right) .
$$

Well, from assumptions (H1)-(H2), we have

$$
\sum_{j=1}^{\infty}\left(\lambda T C_{j}+\lambda \alpha_{j}+\mathbf{E} \int_{0}^{T}\left|g_{j}(s, 0,0)\right|^{2} \mathrm{~d} s\right)<\infty
$$

Consequently, we get,

$$
\mathbf{E}\left(\sup _{0 \leq t \leq T}\left|Y_{t}^{n}-Y_{t}^{m}\right|^{2}+\int_{0}^{T}\left\|Z_{s}^{n}-Z_{s}^{m}\right\|^{2} \mathrm{~d} s\right) \rightarrow 0, \quad \text { as } n, m \rightarrow \infty .
$$

Moreover, from (3.4) together with Hölder’s and Burkholder-Davis-Gundy’s inequalities, we have

$$
\begin{aligned}
\mathbf{E} \sup _{0 \leq t \leq T}\left|K_{t}^{n}-K_{t}^{m}\right|^{2} \leq & 6 \mathbf{E}\left|Y_{0}^{n}-Y_{0}^{m}\right|^{2}+6 \mathbf{E} \sup _{0 \leq t \leq T}\left|Y_{t}^{n}-Y_{t}^{m}\right|^{2}+6 T \mathbf{E} \int_{0}^{T}\left|f\left(s, Y_{s}^{n}, Y_{s}^{n}\right)-f\left(s, Y_{s}^{m}, Z_{s}^{m}\right)\right|^{2} \mathrm{~d} s \\
& +6 \sum_{j=1}^{m} \mathbf{E} \int_{0}^{T}\left|g_{j}\left(s, Y_{s}^{n}, Y_{s}^{n}\right)-g_{j}\left(s, Y_{s}^{m}, Z_{s}^{m}\right)\right|^{2} \mathrm{~d} s+6 \sum_{j=m+1}^{n} \mathbf{E} \int_{0}^{T}\left|g_{j}\left(s, Y_{s-}^{n}, Z_{s}^{n}\right)\right|^{2} \mathrm{~d} s \\
& +6 \mathbf{E} \int_{0}^{T}\left\|Z_{s}^{n}-Z_{s}^{m}\right\|^{2} \mathrm{~d} s,
\end{aligned}
$$

which, together with assumption (H2) and (3.7), provides

$$
\underset{0 \leq t \leq T}{\mathbf{E} \sup _{t}\left|K_{t}^{n}-K_{t}^{m}\right|^{2} \rightarrow 0, \quad \text { as } n, m \rightarrow \infty .}
$$

Consequently, $\left(Y^{n}, Z^{n}, K^{n}\right)$ is a Cauchy sequence in $\mathcal{S}_{\mathcal{F}}^{2} \times \mathcal{P}_{\mathcal{F}}^{2}\left(\ell^{2}\right) \times \mathcal{A}_{\mathcal{F}}^{2}$ which is a Banach space. Therefore, there exists a process $(Y, Z, K) \in \mathcal{S}_{\mathcal{F}}^{2} \times \mathcal{P}_{\mathcal{F}}^{2}\left(\ell^{2}\right) \times \mathcal{A}_{\mathcal{F}}^{2}$, such that

$$
\mathbf{E}\left(\sup _{0 \leq t \leq T}\left|Y_{t}^{n}-Y_{t}\right|^{2}+\int_{0}^{T} \| Z_{s}^{n}-\left.Z_{s}\right|^{2} \mathrm{~d} s+\sup _{0 \leq t \leq T}\left|K_{t}^{n}-K_{t}\right|^{2}\right) \rightarrow 0, \quad \text { as } n \rightarrow \infty .
$$

Now, let us show that the process $(Y, Z, K) \in \mathcal{S}_{\mathcal{F}}^{2} \times \mathcal{P}_{\mathcal{F}}^{2}\left(\ell^{2}\right) \times \mathcal{A}_{\mathcal{F}}^{2}$ satisfies our Equation (1.2). From Cauchy- 
Schwarz inequality, together with (H2), we have

$$
\mathbf{E}\left(\left|\int_{t}^{T} f\left(s, Y_{s}^{n}, Z_{s}^{n}\right) \mathrm{d} s-\int_{t}^{T} f\left(s, Y_{s}, Z_{s}\right) \mathrm{d} s\right|^{2}\right) \leq C T \mathbf{E}\left(T \sup _{0 \leq t \leq T}\left|Y_{t}^{n}-Y_{t}\right|^{2}+\int_{0}^{T}\left\|Z_{s}^{n}-Z_{s}\right\|^{2} \mathrm{~d} s\right) \rightarrow 0, \text { as } n \rightarrow \infty .
$$

Also, by Burkhölder-Davis-Gundy’s inequality, we get

$$
\mathbf{E}\left(\sup _{0 \leq t \leq T}\left|\sum_{i=1}^{\infty}\left(\int_{t}^{T} Z_{s}^{n(i)} \mathrm{d} H_{s}^{(i)}-\int_{t}^{T} Z_{s}^{(i)} \mathrm{d} H_{s}^{(i)}\right)\right|\right) \leq c \mathbf{E} \int_{0}^{T}\left\|Z_{s}^{n}-Z_{s}\right\|^{2} \mathrm{~d} s \rightarrow 0, \text { as } n \rightarrow \infty
$$

and

$$
\begin{aligned}
& \mathbf{E}\left(\sup _{0 \leq t \leq T}\left|\sum_{j=1}^{n} \int_{t}^{T} g_{j}\left(s, Y_{s}^{n}, Z_{s}^{n}\right) \overleftarrow{\mathrm{d} B}_{s}^{j}-\sum_{j=1}^{\infty} \int_{t}^{T} g_{j}\left(s, Y_{s}, Z_{s}\right) \overleftarrow{\mathrm{d} B}_{s}^{j}\right|\right) \\
& \leq c \mathbf{E}\left(\sum_{j=1}^{n} \int_{0}^{T}\left|g_{j}\left(s, Y_{s}^{n}, Z_{s}^{n}\right)-g_{j}\left(s, Y_{s}, Z_{s}\right)\right|^{2} \mathrm{~d} s+\sum_{j=n+1}^{\infty} \int_{0}^{T}\left|g_{j}\left(s, Y_{s}, Z_{s}\right)\right|^{2} \mathrm{~d} s\right) .
\end{aligned}
$$

Now, from (H1)-(H2) and the fact that $(Y, Z) \in \mathcal{S}_{\mathcal{F}}^{2} \times \mathcal{P}_{\mathcal{F}}^{2}\left(\ell^{2}\right)$, we have

$$
\sum_{j=1}^{\infty} \mathbf{E} \int_{0}^{T}\left|g_{j}\left(s, Y_{s}, Z_{s}\right)\right|^{2} \mathrm{~d} s \leq 2 T\left(\sum_{j=1}^{\infty} C_{j}\right) \mathbf{E} \sup _{0 \leq t \leq T}\left|Y_{t}\right|^{2}+2\left(\sum_{j=1}^{\infty} \alpha_{j}\right) \mathbf{E} \int_{0}^{T}\left\|Z_{s}\right\|^{2} \mathrm{~d} s+2 \sum_{j=1}^{\infty} \mathbf{E} \int_{0}^{T}\left|g_{j}(s, 0,0)\right|^{2} \mathrm{~d} s<\infty,
$$

which implies that

$$
\sum_{j=n+1}^{\infty} \mathbf{E} \int_{0}^{T}\left|g_{j}\left(s, Y_{s}, Z_{s}\right)\right|^{2} \mathrm{~d} s \rightarrow 0, \text { as } n \rightarrow \infty
$$

Moreover,

$$
\begin{aligned}
& \mathbf{E}\left(\sum_{j=1}^{n} \int_{0}^{T}\left|g_{j}\left(s, Y_{s}^{n}, Z_{s}^{n}\right)-g_{j}\left(s, Y_{s}, Z_{s}\right)\right|^{2} \mathrm{~d} s\right) \\
& \leq \mathbf{E}\left(\sum_{j=1}^{\infty} C_{j} T \sup _{0 \leq t \leq T}\left|Y_{t}^{n}-Y_{t}\right|^{2}+\alpha \int_{0}^{T}\left\|Z_{s}^{n}-Z_{s}\right\|^{2} \mathrm{~d} s\right) \rightarrow 0, \text { as } n \rightarrow \infty .
\end{aligned}
$$

Therefore,

$$
\mathbf{E}\left(\sup _{0 \leq t \leq T}\left|\sum_{j=1}^{n} \int_{t}^{T} g_{j}\left(s, Y_{s}^{n}, Z_{s}^{n}\right) \overleftarrow{\mathrm{d} B}_{s}^{j}-\sum_{j=1}^{\infty} \int_{t}^{T} g_{j}\left(s, Y_{s}, Z_{s}\right) \overleftarrow{\mathrm{d} B}_{s}^{j}\right|\right) \rightarrow 0 \text {, as } n \rightarrow \infty .
$$

On the other hand, from the result of Saisho [6] (see p. 465), we have

$$
\int_{0}^{T}\left(Y_{s}^{n}-S_{s}\right) \mathrm{d} K_{s}^{n} \rightarrow \int_{0}^{T}\left(Y_{s}-S_{s}\right) \mathrm{d} K_{s} \quad \mathbf{P}-\text { a.s., } \quad \text { as } n \rightarrow \infty .
$$

Finally, passing to the limit in (3.2), we conclude that $(Y, Z, K)$ is a solution of (1.2).

(Uniqueness.) Let $\left(Y^{i}, Z^{i}, K^{i}\right)(i=1,2)$ be two solutions of (1.2).

Applying Itô's formula to $\mathrm{e}^{\beta t}\left|Y_{t}^{1}-Y_{t}^{2}\right|^{2}$, we get

$$
\begin{aligned}
& \mathrm{e}^{\beta t}\left|Y_{t}^{1}-Y_{t}^{2}\right|^{2}+\beta \int_{t}^{T} \mathrm{e}^{\beta s}\left|Y_{s}^{1}-Y_{s}^{2}\right|^{2} \mathrm{~d} s \\
& =2 \int_{t}^{T} \mathrm{e}^{\beta s}\left(Y_{s}^{1}-Y_{s}^{2}\right)\left(f\left(s, Y_{s-}^{1}, Z_{s}^{1}\right)-f\left(s, Y_{s-}^{2}, Z_{s}^{2}\right)\right) \mathrm{d} s \\
& \quad+2 \sum_{j=1}^{\infty} \int_{t}^{T} \mathrm{e}^{\beta s}\left(Y_{s}^{1}-Y_{s}^{2}\right)\left(g_{j}\left(s, Y_{s-}^{1}, Z_{s}^{1}\right)-g_{j}\left(s, Y_{s-}^{2}, Z_{s}^{2}\right)\right) \mathrm{d} B_{s}^{j}+2 \int_{t}^{T} \mathrm{e}^{\beta s}\left(Y_{s}^{1}-Y_{s}^{2}\right)\left(\mathrm{d} K_{s}^{1}-\mathrm{d} K_{s}^{2}\right) \\
& \quad-2 \sum_{i=1}^{\infty} \int_{t}^{T} \mathrm{e}^{\beta s}\left(Y_{s}^{1}-Y_{s}^{2}\right)\left(Z_{s}^{1(i)}-Z_{s}^{2(i)}\right) \mathrm{d} H_{s}^{(i)}+\sum_{j=1}^{\infty} \int_{t}^{T} \mathrm{e}^{\beta s}\left|g_{j}\left(s, Y_{s-}^{1}, Z_{s}^{1}\right)-g_{j}\left(s, Y_{s-}^{2}, Z_{s}^{2}\right)\right|^{2} \mathrm{~d} s \\
& \quad-\sum_{i, j=1}^{\infty} \int_{t}^{T} \mathrm{e}^{\beta s}\left(Z_{s}^{1(i)}-Z_{s}^{2(i)}\right)\left(Z_{s}^{1(j)}-Z_{s}^{2(j)}\right) \mathrm{d}\left[H^{(i)}, H^{(j)}\right]_{s} .
\end{aligned}
$$


Taking expectation in both side of (3.10) and noting that $\int_{t}^{T}\left(Y_{s}^{1}-Y_{s}^{2}\right)\left(\mathrm{d} K_{s}^{1}-\mathrm{d} K_{s}^{2}\right) \leq 0$, we have

$$
\begin{aligned}
& \mathbf{E}\left(\mathrm{e}^{\beta t}\left|Y_{t}^{1}-Y_{t}^{2}\right|^{2}+\beta \int_{t}^{T} \mathrm{e}^{\beta s}\left|Y_{s}^{1}-Y_{s}^{2}\right|^{2} \mathrm{~d} s+\int_{t}^{T} \mathrm{e}^{\beta s}\left\|Z_{s}^{1}-Z_{s}^{2}\right\|^{2} \mathrm{~d} s\right) \\
& \leq 2 \mathbf{E} \int_{t}^{T} \mathrm{e}^{\beta s}\left(Y_{s}^{1}-Y_{s}^{2}\right)\left(f\left(s, Y_{s-}^{1}, Z_{s}^{1}\right)-f\left(s, Y_{s-}^{2}, Z_{s}^{2}\right)\right) \mathrm{d} s \\
& +\sum_{j=1}^{\infty} \mathbf{E} \int_{t}^{T} \mathrm{e}^{\beta s}\left|g_{j}\left(s, Y_{s-}^{1}, Z_{s}^{1}\right)-g_{j}\left(s, Y_{s-}^{2}, Z_{s}^{2}\right)\right|^{2} \mathrm{~d} s .
\end{aligned}
$$

Using again Young's inequality $\left(2 a b \leq \frac{2 C}{1-\alpha} a^{2}+\frac{1-\alpha}{2} b^{2}\right)$ and assumption (H2), we obtain,

$$
\begin{aligned}
& \mathbf{E}\left(\mathrm{e}^{\beta t}\left|Y_{t}^{1}-Y_{t}^{2}\right|^{2}+\beta \int_{t}^{T} \mathrm{e}^{\beta s}\left|Y_{s}^{1}-Y_{s}^{2}\right|^{2} \mathrm{~d} s+\int_{t}^{T} \mathrm{e}^{\beta s}\left\|Z_{s}^{1}-Z_{s}^{2}\right\|^{2} \mathrm{~d} s\right) \\
& \leq\left(\frac{2 C}{1-\alpha}+\sum_{j=1}^{\infty} C_{j}+\frac{1-\alpha}{2}\right) \mathbf{E} \int_{t}^{T} \mathrm{e}^{\beta s}\left|Y_{s}^{1}-Y_{s}^{2}\right|^{2} \mathrm{~d} s+\frac{1+\alpha}{2} \mathbf{E} \int_{t}^{T} \mathrm{e}^{\beta s}\left\|Z_{s}^{1}-Z_{s}^{2}\right\|^{2} \mathrm{~d} s
\end{aligned}
$$

Choosing $\beta>\frac{2 C}{1-\alpha}+\sum_{j=1}^{\infty} C_{j}+\frac{1-\alpha}{2}$, we have $Y_{t}^{1}=Y_{t}^{2}$, a.e., for all $t \in[0, T]$. So, we have $Z_{t}^{1}=Z_{t}^{2}$, a.e., for all $t \in[0, T]$.

On the other hand, since,

$$
\begin{aligned}
K_{t}^{1}-K_{t}^{2}= & \left(Y_{0}^{1}-Y_{0}^{2}\right)-\left(Y_{t}^{1}-Y_{t}^{2}\right)-\int_{0}^{t}\left(f\left(s, Y_{s-}^{1}, Z_{s}^{1}\right)-f\left(s, Y_{s-}^{2}, Z_{s}^{2}\right)\right) \mathrm{d} s \\
& +\sum_{j=1}^{\infty} \int_{0}^{t}\left(g_{j}\left(s, Y_{s-}^{1}, Z_{s}^{1}\right)-g_{j}\left(s, Y_{s-}^{2}, Z_{s}^{2}\right)\right) \mathrm{d} B_{s}^{j}-\sum_{i=1}^{\infty} \int_{0}^{t}\left(Z_{s}^{1(i)}-Z_{s}^{2(i)}\right) \mathrm{d} H_{s}^{(i)}, t \in[0, T],
\end{aligned}
$$

we have $K_{t}^{1}=K_{t}^{2}$, a.e., for all $t \in[0, T]$. Then, we complete the proof.

\section{References}

[1] Ren, Y. (2010) Reflected Backward Doubly Stochastic Differential Equations Driven by a Lévy Process. C. R. Acad. Sci. Paris, Ser. I, 348, 439-444.

[2] Yan, J., He, S. and Wang, J. (1995) Semimartingale and Stochastic Analysis. Scientific Press, Beijing,

[3] Duan, P.J., Ren, M. and Fei, S.L. (2013) Reflected Backward Stochastic Differential Equations Driven by Countable Brownian Motions. Journal of Applied Mathematics, 2013, Article ID: 729636.

[4] Owo, J.-M. (2015) Reflected Backward Stochastic Differential Equations Driven by Countable Brownian Motions with Continuous Coefficients. Electronic Communications in Probability, 20, 1-11. http://dx.doi.org/10.1214/ECP.v20-3771

[5] Nualart, D. and Schoutens, W. (2001) Backward Stochastic Differential Equations and Feynman-Kac Formula for Lévy Processes with Applications in Finance. Bernoulli, 7, 761-776. http://dx.doi.org/10.2307/3318541

[6] Saisho, Y. (1987) SDE for Multidimensional Domains with Reflecting Boundary. Probability Theory and Related Fields, 74, 455-477. http://dx.doi.org/10.1007/BF00699100 